\title{
Analysis of Energy Consumption and Lifetime of Heterogeneous Wireless Sensor Networks
}

\author{
Enrique J. Duarte-Melo, Mingyan Liu \\ EECS, University of Michigan, Ann Arbor \\ \{ejd, mingyan\}@eecs.umich.edu
}

\begin{abstract}
The paper examines the performance as well as energy consumption issues of a wireless sensor network providing periodic data from a sensing field to a remote receiver. The sensors are assumed to be randomly deployed. We distinguish between two types of sensor organizations, one with a single layer of identical sensors (homogeneous) and one with an additional overlay of fewer but more powerful sensors (heterogeneous). We formulate the energy consumption and study their estimated lifetime based on a clustering mechanism with varying parameters related to the sensing field, e.g., size, and distance. We quantify the optimal number of clusters based on our model and show how to allocate energy between different layers.
\end{abstract}

\section{INTRODUCTION}

The rapid development in small, low-power, low-cost microelectronic and micro-electromechanical (MEMs) sensor technology [1] along with the advances in wireless technology have enabled wireless sensors to be deployed in large quantities to form wireless sensor networks for a wide variety of purposes, e.g., image formation of a target field, intrusion detection, surveillance and monitoring. Such deployment can be random (e.g., dropped from an airplane) or planned and calculated (e.g., installing fire alarm sensors in a building).

Communications in a wireless sensor network occur in different ways depending on the underlying application or mission of the network. In general, there are three types of communications: clock-driven, event-driven and query-driven. Clockdriven communication takes place where sensors gather and send data at constant periodic intervals. Such periodic transmission can be destined for a certain receiver or collector. Combining data from all sensors generates "snapshots" of the field that is being sensed. Over time these snapshots produce temporal and spatial information about the field, e.g., acoustic, seismic, meteorological. Query-driven or event-driven communications are triggered by certain events or queries. These different types of communication can appear in combination in a sensor network.

In all these cases the lifetime of a sensor and the lifetime of the network, which directly determines the duration of the sensing task, is limited by the amount of energy each sensor has. Therefore when we examine these networks, efficient use of energy is a primary concern. While it is tempting to simply apply existing research in wireless networks to sensor networks, sensor networks have enough particular characteristics and challenges to justify their specific study [2]. Sensor networks have been quite extensively studied in the past few years, see for example [3], [4], [1], [5], and [6].

This project is funded by NSF ITR grant number ANI-0112801.
To the best of our knowledge, almost all of the existing work focuses on sensor networks that consist of identical sensors with equal capacity in terms of sensing, computation, communication, and power. Consequently we consider this type of sensor networks homogeneous. The possibility of working with more than one type of sensors within a same network is mentioned in [7], but without an in depth study of this possibility. We observe that the manufacturing and thus the functionality of a sensor is generally very application-specific. We anticipate different special purpose sensors can be used to form a single sensor network to perform more comprehensive tasks, e.g., some sensors collect image data, some sensors collect audio signal, some sensors have more processing capability, some sensors have more power, and so on. This results in a heterogeneous sensor network that can have a variety of compositions of sensors. Many organizational and communication issues arise with such a structure.

In this paper we examine one of the simplest such heterogeneous scenarios - in which sensors are equipped with different battery power - in a clock-driven sensor network. In particular, we consider a field randomly deployed with sensors that gather data and transmit it back to a remote receiver, which is assumed to be located away from the sensing field. Such a scenario is motivated by applications in which data is desired from a hostile environment,such as a volcano or a swamp, where sensors are likely to be deployed in an unmanned manner.Under such situations sensing data will be collected, analyzed and interpreted at a more accessible location. The main issue we are interested in is how to maximize the lifetime of such a sensor network for a given amount of energy, or equivalently, how to retrieve the same data using the least amount of energy. In this paper we define the lifetime of a sensor network as the expected lifetime of any given sensor in the network. In a densely deployed sensor network this definition can be easily extended to be the time until a certain percentage of the sensors died. Thus in prolonging the lifetime of a sensor network, it is important to balance the power depletion from one sensor to another.

There are different ways of collecting the sensing data. The simplest is direct transmission, where each sensor directly sends gathered information to the remote receiver independent of each other. This approach is mentioned and used for comparison in [3]. It does not require any communication between sensors. This approach has an inherent scalability problem considering the fact that this is a many-to-one communication where the number of sensors can be potentially huge. In addition, this also puts a limit on how far away from the sensing field the remote 
collector can be since each sensor will need to be able to reach the collector. A second approach is via multi-hop routing, which has been extensively studied for both generic ad hoc routing networks as well as wireless sensor networks, e.g., [8], [9] and [4]. Such routing protocols can be designed to realize different goals, e.g., minimize energy consumption. However, these protocols are typically evaluated assuming a random traffic pattern, and it is not clear how they would perform under the scenario where communications are mostly all-to-one or all-to-few (i.e., there can be a small number of collectors). Further investigation on using multi-hop routing within this context is part of our ongoing research.

A third approach is clustering, where sensors form clusters dynamically with neighboring sensors. One of the sensor in the cluster will be elected cluster head and be responsible for relaying data from each sensor in the cluster to the remote receiver/collector. This approach localizes traffic and can potentially be more scalable. In addition, the cluster heads naturally become points where data fusion and data compression can occur considering the potential correlation among data from neighboring sensors. Since the cluster heads will inevitably consume more energy and thus die sooner than other sensors, methods of dynamically changing cluster heads are preferred so that the use of energy can be spread as evenly as possible among all sensors, see for example [3].

In this paper we will focus on the clustering approach and examine the use of a heterogeneous structure where some sensors carry more power than others, and thus naturally become cluster heads. In this case the field is first deployed with a number of type-I sensors, and then deployed with an overlay of type-II sensors, presumably more powerful but fewer in number. We will call these overlay sensors and the first layer sensors normal sensors in subsequent discussion. The overlay sensors can potentially have more processing capability and communication capability in addition to having more energy. We formulate the energy consumption for the heterogeneous case and estimate its lifetime with varying parameters related to the sensing field, e.g., size, distance, and so on. We quantify the optimal number of clusters based on our model and show how to allocate the energy between the overlay sensors and normal sensors. We will also relate these results to the homogeneous case.

The remainder of the paper is organized as follows. First we present the network model under consideration and related assumptions in Section II. Section III provides a mathematical formulation that attempts to estimate the average lifetime of a sensor network. We also discuss related energy allocation issues and the optimal number of clusters in this section. We conclude the paper with a summary and discussion of future work in Section IV.

\section{Network MOdEL AND AsSUMPtions}

Consider a square sensing field with each side measuring $L$ meters. The coordinates of the field are as shown in Figure 1, where crosses represent overlay sensors, and circles represent normal sensors. All data collected by the sensors is to be sent to a receiver/collector located outside the sensing field. We will use the terms receiver and collector interchangeably in subsequent discussions. The collector is located at $(0,-D)$, and is thus $D$ meters away from the sensing field. This location is assumed to be fixed. We assume all sensors are aware of the location of the receiver via some type of pre-configuration or selfconfiguration. There is a total of $n$ normal sensors in the field.

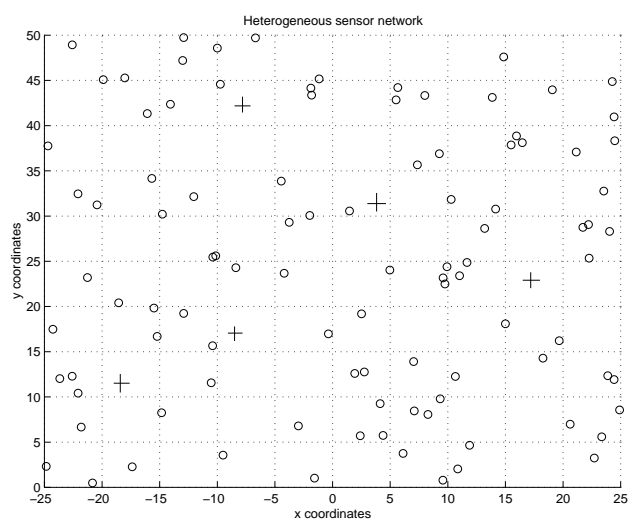

Fig. 1. Heterogeneous Network

They are assumed to be uniformly distributed within the field. In addition to that, there are $R \cdot q$, (where $R, q>1$ ) overlay sensors in the field, also randomly deployed. On average only $q$ overlay sensors are active at any given time, i.e., on average there are $q$ clusters. These overlay sensors will take turns (in a way described below) being cluster heads. The reason for such redundancy is that a very uneven topology due to randomness in deploying these overlay sensors could lead to rapid energy depletion of a particular overlay sensor and in turn, this can have an adverse effect on the lifetime of the network. If we deploy more overlay sensors than needed and then randomly choosing a subset to be active periodically, we may "even out" the random effect of deployment.

We assume that sensed data is collected in a periodic manner, and each such period is defined as a round. This period that we have named round consists of the sensing of the data and the transmission of one packet containing the data sensed to the cluster head. The round also includes the relay of that packet and the packet of each sensor in the cluster to the collector. Furthermore, we assume that each sensor has a constant amount of raw data to send, including the overlay sensors if active. Thus every sensor, in every round, has $b_{s}$ bits to send. A round starts with each overlay sensor dynamically deciding whether it will be a cluster head in the current round. If so it broadcasts its presence to the normal sensors and starts receiving data from the sensors that have decided to become part of its cluster. Normal sensors decide to which cluster they wish to belong based on the strength of the signal from the broadcast. It is assumed that the stronger the signal, the closer the head is and therefore the head with the strongest signal is chosen. If an overlay sensor decides not to be a cluster head for the current round, it goes to sleep for the duration of the round. Once the data from all the sensors within the cluster is gathered, it is relayed to the collector. This marks the end of a round the beginning of the next round. By speci- 
fying that an overlay sensor is active once and only once every $R$ rounds, we ensure that on average there are $q$ clusters in the network. This method is borrowed from [3], with the only difference being that in [3] such a decision is made by homogeneous sensors, and here it is made only by overlay sensors.

We assume some form of MAC is used within each cluster, see for example [10]. Some form of MAC is also used between multiple cluster heads and the remote collector, but the communication between cluster heads and the remote collector takes place in a different channel than that between normal sensors.

We adopt the following energy model [3]:

Energy spent in transmission $=e_{d} b d^{\alpha}+e_{t} b$;

Energy spent in reception $=e_{l} b$; and

Energy spent sensing $=e_{s} b$, where $e_{d}$ is the energy dissipated per bit per $m^{2}$ and is chosen to be $100 \times 10^{-12}, e_{t}$ is the energy spent by transmission circuitry per bit and is chosen to be $50 \mathrm{x}$ $10^{-9}, e_{l}$ is the energy spent by reception circuitry per bit and is chosen to be $50 \times 10^{-9}, e_{s}$ is the energy spent sensing per bit and is chosen to be $50 \times 10^{-9}, b$ is number of bits to transmit or receive, $d$ is the distance from transmitter to receiver and $\alpha$ is a constant $\geq 2$ which depends on the attenuation the signal will suffer in that environment

In our analysis we will use the common values of $\alpha=2$ and $\alpha=4$. In this paper we limit ourselves to only consider the energy consumed in communication, as illustrated above. Further study is needed to also take into account energy consumed in data processing, etc. Throughout this paper we do not take into account the energy consumed at the remote receiver, which is assumed to have less stringent energy constraint. The focus is therefore on the limited energy available to the sensors which rely on batteries.

\section{ANALYSIS AND NUMERICAL RESUlTS}

In this section we analyze the cluster formation approach in a heterogeneous network. This analysis seeks to estimate the point in time when a given sensor in the network is expected to run out of energy. Setting our objective to maximizing this value, we also illustrate how energy should be allocated between the overlay sensors and the normal sensors, and the optimal number of clusters.

\section{A. Estimation of network lifetime}

Following the procedure described in the previous section we can guarantee that every overlay sensor will be cluster head once and only once every $R$ rounds, and that the average number of clusters in the network is $q$. Below we will focus on the expected energy consumed every $R$ rounds.

Let $W_{o}$ and $W_{s}$ denote the energy consumed by an overlay sensor/cluster head, and a normal sensor (first layer), respectively. Let $S$ be the random variable denoting the distance from the sensor to the cluster head and $Z=d^{2}$ be the random variable denoting the squared distance from the sensor to the receiver. The expected energy spent every $R$ rounds by a cluster head is

$$
W_{o}=K_{1}+\left(K_{2}+A_{1} E\left[d^{\alpha}\right]\right) C_{o}(q)
$$

where $K_{1}, K_{2}$ and $A_{1}$ are constants which depend in the number of bits in the packets, the energy used by the circuitry for every bit, the number of sensors, the number of heads and the size of the field. Their calculations are given in Appendix. $C_{o}(q)$ is the expected number of transmissions needed to achieve a successful transmission from the cluster head to the remote collector as a function of active cluster heads. The exact expression of this function will depend on the MAC scheme used between the cluster heads and the collector. Appropriate MAC schemes are beyond the scope of this paper. For an evaluation of different MAC schemes see for example [10].

Similarly, the expected energy spent by a normal sensor in the heterogeneous case every $R$ rounds is

$$
W_{s}=K_{3}+\left(K_{4}+A_{2} E\left[S^{\alpha}\right]\right) C_{s}\left(\frac{n}{q}\right)
$$

where $K_{3}, K_{4}$ and $A_{2}$ are again constants given in the Appendix. Again $C_{s}(n / q)$ is the expected number of transmissions needed to achieve a successful transmission within a cluster as a function of the average size of cluster. This function depends on the MAC used within each cluster.

The sensors are assumed to be uniformly distributed both in $x$ and $y$. With a few calculations we can show that

$$
\begin{aligned}
E[Z] & =E\left[d^{2}\right]=E\left[x^{2}+(y+D)^{2}\right] \\
& =\frac{1}{3}\left(\frac{L}{2}\right)^{2}+\frac{L^{2}}{3}+D L+D^{2}
\end{aligned}
$$

and

$$
\begin{aligned}
E\left[Z^{2}\right] & =E\left[d^{4}\right]=E\left[\left(x^{2}+(y+D)^{2}\right)^{2}\right] \\
& =\frac{193 L^{4}}{720}+\frac{7 D L^{3}}{6}+\frac{25 D^{2} L^{2}}{12}+2 D^{3} L+D^{4}
\end{aligned}
$$

Similarly we can show that the pdf of $S$ is

$$
f_{S}(s)=q\left(\frac{\sqrt{2}}{L}-\frac{s}{L^{2}}\right)\left(1-\frac{s \sqrt{2}}{L}+\frac{s^{2}}{2 L^{2}}\right)^{q-1}
$$

This is used to calculate the expected value of $S^{\alpha}$, which is:

$$
E\left[S^{\alpha}\right]=\int_{0}^{2 L^{2}}\left[q s^{\alpha}\left(\frac{\sqrt{2}}{L}-\frac{s}{L^{2}}\right)\left(1-\frac{s \sqrt{2}}{L}+\frac{s^{2}}{2 L^{2}}\right)^{q-1}\right] d s
$$

Note that the above results ((1)-(4)) can easily be extrapolated to model direct transmission approach, since it depends only on a few constants and the random variable $Z$. They can also be extended to model the homogeneous case. In fact since the energy used by the sensors when they act as cluster head in a homogeneous network is the same used by overlay sensors in a heterogeneous network in a given round, the overall expected lifetime of the network is the same, given same amount of total energy. Note that in the homogeneous case every sensor has to be equipped with enough power to reach the collector, while in the heterogenous case this is not needed. 
The above equations can also help us determine a reasonable energy allocation for the heterogeneous case, as we show below. The key question is that since we have different types of sensors, given a fixed budget and a fixed task, how much energy with which the normal sensors and the overlay sensors should be equipped, respectively. One possible criteria is to let the two types of sensors have the same targeted life time. This criteria would apply to scenarios where sensors are not considered retrievable or reusable. Thus by balancing energy allocation, by using (1) and (2), we can maximize the network life time for a given fixed amount of energy. Let $\phi$ be the energy allocated to the overlay sensors and $\beta$ be the energy allocated to the normal sensors.

$$
\frac{\beta}{K_{3}+\left(K_{4}+A_{2} E\left[S^{\alpha}\right]\right) C_{s}\left(\frac{n}{q}\right)}=\frac{\phi}{K_{1}+\left(K_{2}+A_{1} E\left[d^{\alpha}\right]\right) C_{o}(q)}
$$

From there we get the relation:

$$
\frac{\beta}{\phi}=\frac{K_{1}+\left(K_{2}+A_{1} E\left[d^{\alpha}\right]\right) C_{o}(q)}{K_{3}+\left(K_{4}+A_{2} E\left[S^{\alpha}\right]\right) C_{s}\left(\frac{n}{q}\right)}
$$

Following this, both the overlay and the normal sensors in the network should last

$$
R_{s}=R\left(\frac{\beta}{K_{3}+\left(K_{4}+A_{2} E\left[S^{\alpha}\right]\right) C_{s}\left(\frac{n}{q}\right)}\right)
$$

number of rounds.

It is not easy to draw conclusions directly from these equations for the lack of a closed form solution in some cases. However by using a few numerical methods to solve the equations under given circumstances, we can gain some insight. All our calculations are done in Matlab. For simplicity reasons, we will assume that perfect scheduling is achieved at the MAC layer and therefore $C_{o}(q)=C_{s}(n / q)=1$. However, the framework of the analysis does not change as the MAC scheme changes with a different function.

\section{B. Optimal number of clusters}

Note that $E\left[S^{\alpha}\right]$ depends on $q$, the number of clusters. This suggests that we could use (7) to determine how many cluster heads are needed.

The plots of Figures 2, 3 and 4 are obtained assuming the perfect scheduling mentioned before, data packets of 1024 bits and control packets of 128 bits. We also assume that the total energy of the network is bounded by 50 joules in each case. This number is fairly arbitrary, but does not affect the behavior of the network. For a different value the plots in the Figures 2, 3 and 4 will simply be scaled. Without such a fixed bound, the extra overlay sensors would result in added energy to the network and thus increased lifetime. In each of these cases, i.e., with a different number of clusters, the amount of energy carried by a normal sensor and an overlay sensor is determined by (6).

In these experiments we set the total number of sensors (both types) to be fixed at 100 . The expected number of active overlay sensors will be $q$, and $100-q$ is the number of normal sensors.

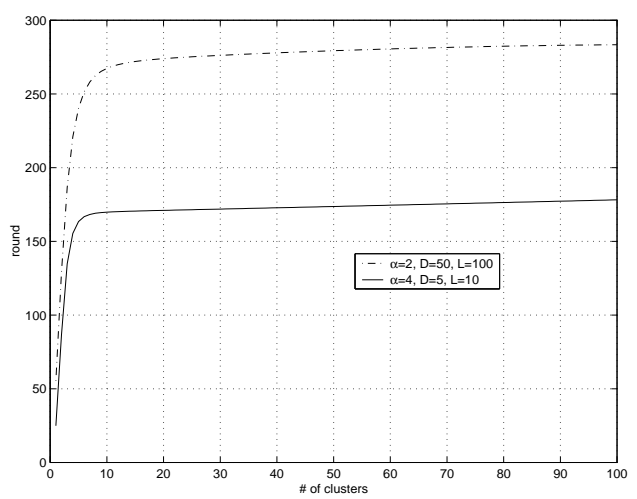

Fig. 2. $\alpha=2, L=50, D=100$ and $\alpha=4, L=5, D=10$

We let $q$ vary from 1 to 100 . In other words, the average number of active sensors (normal plus overlay) remains fixed, while the average number of clusters increases. Since an overlay sensor also has normal sensing capability, by doing so we fix the amount of sensing data from one scenario to another. In addition, under such a setup, $q=100$ would correspond to direct transmission. Figures 2 through 4 show the expected number of rounds the network can last as a function of number of clusters. In Figure 2 there is a clear knee corresponding to $q$ between 4 and 10 , while the maximum is reached when $q=100$, which represents direct transmission. This is because we have assumed perfect MAC scheduling, and that direct transmission does not involve cluster formation overhead that is incurred periodically otherwise. Intuitively we would like to maximize the life time of the network while minimizing the number of clusters in order to be scalable. Therefore the optimal average number of clusters is determined as the knee in the curve. The exact number for $q$ however varies slightly depending on other parameters such as $L, D$ and $\alpha$.

The change of $\alpha$, or a scale-down in $L$ and $D$ (ratio remains the same) does not change the overall shape of the curves (see Figure 2). An increase in the dimension of the network also does not change the overall shape, see Figure 3. Setting $L=500$ and $\mathrm{D}=1000$ reduces the number of rounds the network can last, but not the location of the knee. Note that in this last case having a large number of clusters no longer means a longer lifetime. The explanation is as follows. In a network of this size $(L=500)$, the distance from sensors to a cluster head increases, i.e., the network becomes more sparse. However, the transmission range needed for broadcast during cluster formation by a cluster head increases even more. Therefore beyond a certain range the increased number of clusters result in higher energy consumption.

Reversing the ratio between $L$ and $D$ results in a very different shape of this curve, see Figure 4. In this case it is not so obvious what the best choice for $q$ would be.

Our explanation is the following. In the cases shown in Figures 2 and 3 the energy spent in propagation is the dominant factor in the total amount of energy consumed, and the amount of energy spent in transmission and reception circuitry is relatively minimal. However, in Figure 4 the distance between the receiver and the field is small enough to allow the energy spent in the 


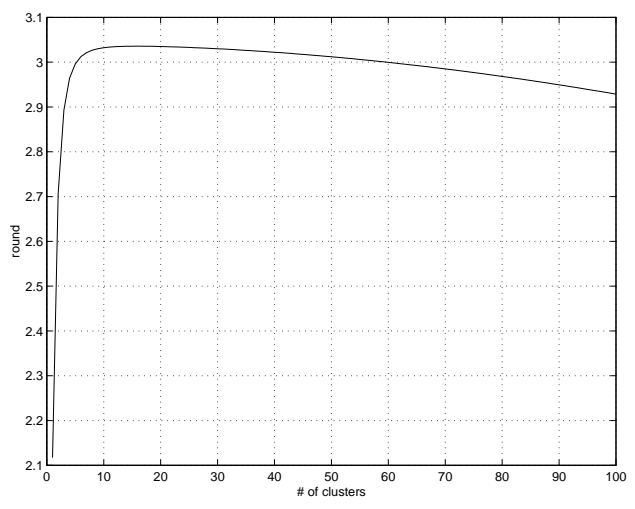

Fig. 3. $\alpha=2, L=500, D=1000$

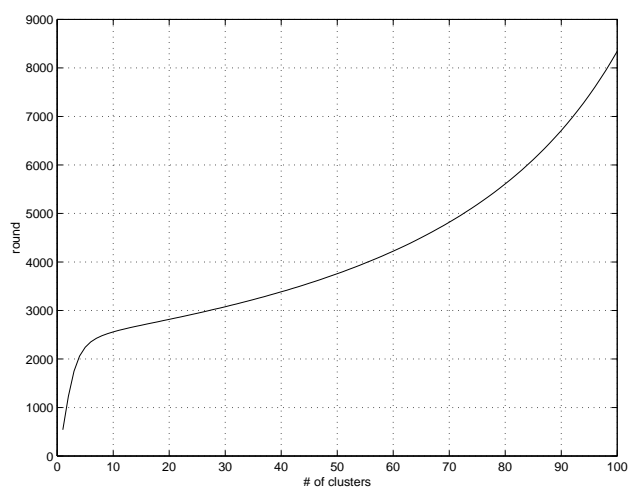

Fig. 4. $\alpha=2, L=5, D=1$

circuitry to become significant. In this case direct transmission becomes a valid choice, assuming that (near) perfect scheduling is possible. Therefore for $q$ to be between 4 and 10 is only recommended for scenarios where the sensing field and the receiver are far away comparing to the size of the sensing field. When the field is large comparing to the distance between the field and the receiver, direct transmission seems to be a good idea and we should choose $q$ as high as the receiver can handle.

\section{Summary and discussions}

In figures presented above, we showed how our analysis can be used to determine the appropriate number of heads in the network. In summary, regardless of whether the network is homogeneous or heterogenous, under the considered parameters, the optimal number of clusters is between 4 and 10 . The exact number varies slightly depending on the size of the field, the location of the receiver, etc. (due to space limit we are not able to show more results). However, when the distance to the receiver is very small comparing to the size of the field, it seems the best choice is to use as many clusters as the receiver can handle.

\section{CONClusion ANd Future Work}

In this paper a constant update or clock-driven sensor network was analyzed with a heterogenous organization. We described how dynamic clusters are formed, presented a way of determining the optimal number of clusters for a given set of parameters, and showed numerically results.

Only a limited number of aspects of a sensor network have been considered here. Future work would explore similar issues in a query-driven and event-driven type of sensor networks. The possibility of several collectors located in different places should also be considered.

Another important issue to be explored is a heterogenous network model where the difference between the sensors is not only the difference in available energy, but also in their processing capabilities, and thus the consideration of energy consumption in data processing (compression, fusion, etc.). Throughout the paper efficient use of the energy was given top priority. In cases where delay and the resolution of the data are just as important, these performance measures should be considered jointly with energy efficiency.

\section{REFERENCES}

[1] A. Chandrakasan, R. Amirtharajah, S. Cho, J. Goodman, G. Konduri, J. Kulik, W. Rabiner, and A. Wang, "Design considerations for distributed microsensor systems," Tech. Rep., Massachusetts Institute of Technology, 2000.

[2] D. Estrin, R. Govindan, J. Heidemann, and S. Kumar, "Next century challenges: Scalable coordination in sensor networks," Tech. Rep., USC/Information Sciences Institute, 2000.

[3] W. R. Heinzelman, A. Chandrakasan, and H. Balakrishnan, "Energyefficient communication protocol for wireless microsensor networks," in Proceedings of the Hawaii International Conference on System Sciences, January 2000.

[4] C. Intanagonwiwat, R. Govidan, and D. Estrin, "Directed diffusion: A scalable and robust communication paradigm for sensor networks," Tech. Rep., USC/Information Sciences Institute, 2000.

[5] W. R. Heinzelman, J. Kulik, and H. Balakrishnan, "Adaptive protcols for infromation dissemination in wireless sensor network," in Fifth ACM/IEEE MOBICOM Conference, Seattle, WA, August 1999.

[6] J. Elson and D. Estrin, "Random, ephimeral transaction identifiers in dynamic sensor networks," Tech. Rep., Department of Computer Science, University of California, Los Angeles; and USC/Information Sciences Institute, 2000.

[7] G. J. Pottie and W. J. Kaiser, "Wireless integrated network sensors," Communications of the ACM, vol. 43, no. 5, May 2000.

[8] Jae-Hwan Chang and Leandros Tassiulas, "Energy conserving routing in wireless ad-hoc networks," IEEE INFOCOM, 2000.

[9] Anastassios Michail and Anthony Ephremides, "Energy efficient routing for connection-oriented traffic in ad-hoc wireless networks," Tech. Rep., Department of Electrical and Computer Engineering and Institute for Systems Research, University of Maryland, 2000.

[10] Enrique J. Duarte-Melo and Mingyan Liu, "Efficient mac for clustered wireless sensor networks," Tech. Rep., Electrical Engineering and Computer Science Department, University of Michigan, Ann Arbor, MI, 2002.

APPENDIX

\begin{tabular}{|c|c|}
\hline Variable Name & Description \\
\hline$b_{b r}$ & Bits sent in broadcast \\
\hline$b_{s}$ & Bits sent per sensor \\
\hline$n$ & Number of sensors \\
\hline$q$ & Number of cluster heads \\
\hline$R$ & as defined in section III.A \\
\hline$M$ & $\frac{n}{q} \in \mathbb{N}$ \\
\hline$B r$ & $\left(b_{b r}\right)\left(e_{d}\right) 2 L^{2}+\left(b_{b r}\right)\left(e_{t}\right)$ \\
\hline$A_{1}$ & $M\left(b_{s}\right)\left(e_{d}\right)$ \\
\hline$K_{1}$ & $B r+(M-1)\left(e_{l}\right)\left(b_{s}\right)+\left(e_{s}\right)\left(b_{s}\right)$ \\
\hline$K_{2}$ & $M\left(e_{t}\right)\left(b_{s}\right)$ \\
\hline$K_{3}$ & $R\left(b_{b r}\right)\left(e_{l}\right)+R\left(b_{s}\right)\left(e_{s}\right)$ \\
\hline$A_{2}$ & $R\left(b_{s}\right)\left(e_{d}\right)$ \\
\hline$K_{4}$ & $R\left(b_{s}\right)\left(e_{t}\right)$ \\
\hline
\end{tabular}

